\title{
A SIMPLE COMPARATIVE BONDING STRENGTH TEST METHOD FOR COMPOSITE RESINS $\dagger$
}

\author{
By Hamdi Mohammed*, D.D.S., M.Sc.D., Ph.D. and E. R. Burrell, D.M.D.**
}

A simple and economical tensile test for measuring bond strength has been developed and utilized to compare the adherence of four composite resins. Specimen preparation is convenient and conditioning treatments can be performed easily. Statistical analysis of the data obtained indicates that the test is useful for comparative adhesion studies.

Adherence between composite resin restorations and cavity walls is important tc inhibit marginal disruption by forces developing during resin hardening and due to temperature fluctuations in the oral cavity. Despite this clinical need, a simple yet reliable tensile test to assess the bond strength of various resin preparations to tooth substance has not been available. While quantitative determination of cement and resin tooth bonding has been reported after tensile loading, ${ }^{17}$ such testing has involved a variety of procedures not generally suitable for widespread comparison of different products, surface pretreatments, and other factors important in determining the adhesive bond strength.

The purpose of this study was to describe a convenient tensile testing method for the assessment of resin-tooth bonding and its application in comparing the adherence of four commercially available composite resins to human enamel and dentin, with and without a bonding agent pretreatment.

\section{Materials and Methods}

Recently extracted human teeth were placed in $0.9 \%$ saline and stored at $6^{\circ} \mathrm{C}$. Individual teeth were mounted in cold curing polyester casting resin. For enamel testing, the labial aspect of incisors was the initial working surface. For dentin testing, occlusal surfaces of third molars were used. An area of enamel or dentin of a diameter greater than $5 \mathrm{~mm}$ was exposed using 120 grit carbimet paper*, and further prepared by unidirectional dry hand sanding to 600 grit for enamel and 400 grit for dentin.

*Professor, Department of Dental Biomaterials College of Dentistry University of Florida

** In the private dental practice, Indianlantic, Florida.

$†$ This study was presented before the 55 th General Meeting of the I.A.D.R., Copenhagen, Denmark.

This study was partially supported by the Kerr Manufacturing Company, Romulus, Michigan.
Specimens were scrubbed with hand soap and a soft bristle toothbrush between various grits.

Enamel surfaces were acid-etched for one minute using the conditioning solution supplied by the manufacturer. Dentin specimen surfaces were not acid-etched. A $5 \mathrm{~mm}$ diameter and $10 \mathrm{~mm}$ deep simulated cavity was formed by a split teflon disk clamped over the test surface which formed the cavity floor. The cavity was filled with freshly mixed resin after application of the intermediate bonding agent where appropriate. An aluminum sleeve with an axially drilled hole was fitted to the cavity margins. A $25 \mathrm{~mm}$ long modified finishing nail with slip fit to the sleeve was pushed into the resin. The sleeve was designed so that the nail head came to rest exactly $1 \mathrm{~mm}$ from the resin/tooth interface when the nail tip was flush with the sleeve top. Perpendicularity of the nail to the specimen surface was assured by means of a shoulder on tile sleeve which mated with the rim of the teflon disk.

•Buehler Ltd., Evanston, Illinois 60204

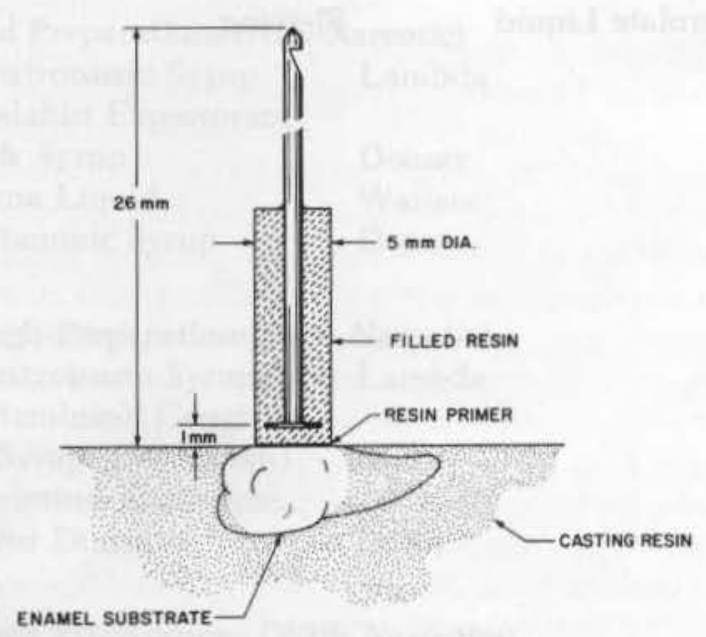

Figure 1-Completed Specimen for Adhesion Testing 
Four resin systems were compared: Two autopolymerizing composite resins (Adaptic* and Concise $^{* *}$ ) with unfilled BIS-GMA bonding agents (Adaptic Bonding Agent* and Concise Enamel Bond System**), one auto-polymerizing composite resin with polycarboxylate bonding agent pretreatment (Simulate $\dagger$ ); and one ultraviolet curing resin (Nuva-Fil $\dagger \dagger$ ) with unfilled BIS-GMA primer (Nuva-Seal $\uparrow \uparrow$ ). All materials were manipulated according to the manufacturer's directions. The bonding agent supplied was applied to the test surface just prior to the mixing of the resin, and the resin was applied to the tooth immediately after mixing. After polymerization of each resin for four minutes, the clamps, sleeve and teflon disk were removed. A completed specimen is illustrated in Figure 1. All specimens were conditioned at $37^{\circ} \mathrm{C}$ and $100 \%$ relative humidity for 24 hours prior to testing.

For ultraviolet curing resins, a button of resin (and bonding agent where appropriate) $5 \mathrm{~mm}$ in diameter and less than $1 \mathrm{~mm}$ thick was formed on the cavity floor. The resin was polymerized for two minutes by placing the ultraviolet activation unit at the rim of the cavity (UV is $9 \mathrm{~mm}$ away from resin surface), the teflon disk was then removed and the button was exposed directly to the ultraviolet light for an additional minute. The total polymerization time of three minutes, one minute greater than manufacturer's recommendation, compensated for the distance created by the thickness of the teflon disk during the first two minutes. The teflon disk was replaced and the remaining of the cavity was filled with an auto-polymerizing resin (Adaptic) preceded by its unfilled resin primer.

Adhesion was evaluated on a Universal mechanical testing machine $\dagger \dagger \dagger$ at a cross head speed of $0.5 \mathrm{~mm}$ per minute. The specimen was mounted in a jig attached to the lower grip through a frictionless universal joint. The specimen was passed through a $7 \mathrm{~mm}$ hole in the restraining plate of the jig and connected to the upper grip by securing the machined nail to an aluminum sleeve at the free end of a suspended $10 \mathrm{~cm}$ long flue chain. This arrangement, shown in Figure 2, assured an applied stress perpendicular to the test surface.

In addition to the above experimental procedure, the variance of proposed test was predetermined by using aluminium as a substrate.

\section{Results}

The present test method yielded a relatively low coefficient of variation of $13.8 \%$ when aluminum disks were used as the substrate.

The mean values for adhesion of the four resins tested on human enamel, when a primer was and was not used is depicted in the table. Each value in

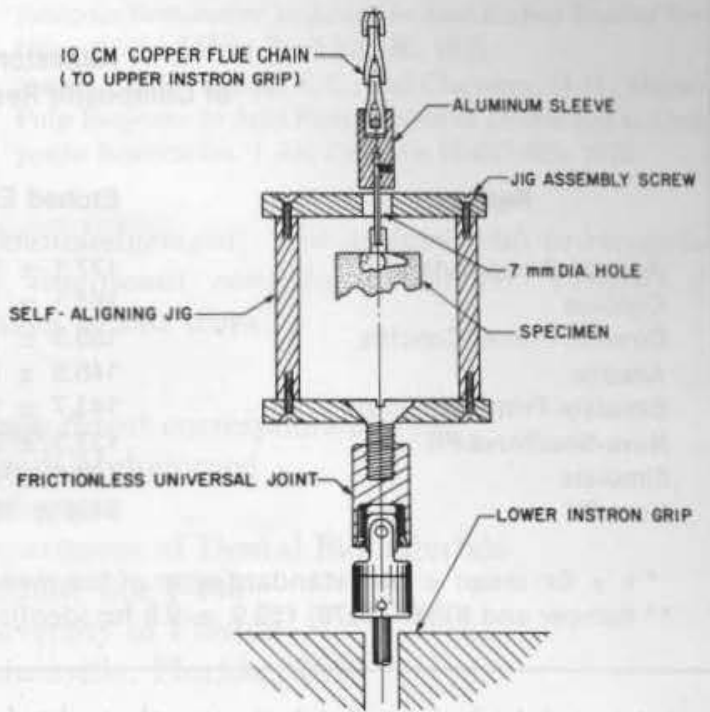

Figure 2-Specimen Assembly Mounted in Testing Machine

the table is the mean of ten specimens. The data was analyzed using a one way analysis of variance. ${ }^{8}$ Since significant differences between the adhesion of various materials was detected at $\mathrm{P}<0.05$, the differences between individual materials were identified by Duncan's multiple range test. ${ }^{9}$ In the table, any two means joined by a line are not significantly different.

The mean adhesion values to human enamel, when a bonding agent is used may be ranked as Adaptic $<$ Concise $<$ Simulate $<$ Nuva-Fil. However, these differences are not statistically significant. The elimination of the primer did not lead to a significant decrease in adhesion for any of the products tested.

Adhesion test data for Adaptic with bonding agent, prepared and conditioned as in the present study obtained, by a test that maintains proper tensile alignment throughout all phases of specimen preparation and testing, ${ }^{7}$ are also included in the table. According to a Student $t$ test, there is no significant difference between this data and that obtained in the present study $(\mathrm{P}<0.05)$.

Results obtained for the adhesion of Simulate and Concise resin systems to unetched and etched enamel surfaces are also included in the table. While differences between products in either condition are not significant, the differences between the etched and unetched condition are highly significant. The ratio of adhesive bond strengths of the

\footnotetext{
"Johnson \& Johnson, New Brunswick, New Jersey 08903

* Minnesota Mining and Mfg. Co., St. Paul, Minnesota 55119

†Kerr Mfg. Co., Romulus, Michigan 48174

††L.D. Caulk Co., Milford, Delaware 19963

$\uparrow \uparrow$ Model 1125, Instron Corp., Bedford, Maine
} 


\section{Resin}

Adaptic Primer/Adaptic
Concise
Concise Primer/Concise
Adaptic
Simulate Primer/Simulate
Nuva-Seal/Nuva-Fil
Simulate

Nuva-Fil
Etched Enamel

$$
\begin{aligned}
& 177.1 \pm 15.1 \cdots \\
& 165.1 \pm 14.4 \\
& 155.3 \pm 16.7 \\
& 146.6 \pm 11.6 \\
& 141.7 \pm 15.0 \\
& 137.1 \pm 21.9 \\
& 127.6 \pm 13.4 \\
& 94.4 \pm 18.2
\end{aligned}
$$

Unetched Enamel

Dentin

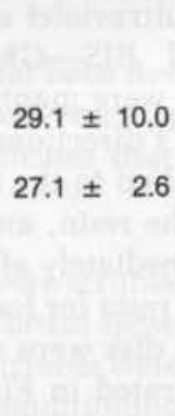

$9.8 \pm 3.0$

$9.9 \pm 1.9$

$6.5 \pm 0.95$

$12.6 \pm 3.0$

* $\mathrm{x} \pm \mathrm{Sx}$; mean \pm one standard error of the mean.

*. Kemper and Kilian (1976) $159.9 \pm 9.5$ for identical conditions.

resin to unetched enamel relative to that obtained when the enamel was etched, is approximately $19 \%$ in each case.

Results obtained for the adhesive strength of the various resins to human dentin is also included in the table. In the absence of a bonding agent pretreatment, all dentin specimens fractured during handling. The bond strength of the resin systems to dentin, relative to that of acid-etched enamel, ranges from 4.5 to $9.2 \%$ when a bonding agent pretreatment was used.

\section{Discussion}

The necessary retentive strength for clinically acceptable restoration longevity has not been clearly established. However, all resin systems exhibited adequate adhesion to enamel, if Bowen's estimate of $49 \mathrm{kgm} / \mathrm{cm}^{2}$ stress developing at the walls of a cavity during the hardening of the resin is considered. ${ }^{10}$

The present data do not show a significant increase in adhesive strength when a bonding agent is employed for the products tested. This is in agreement with previous bending mode adhesion test results," and is consistent with microstructural observations. ${ }^{12}$ Apparently, adherence of composite resin to acid-etched enamel surfaces is not dependent upon the intentional placement of an intermediate film of low viscosity, unfilled resin.

From a retentive standpoint, no advantages seems to accrue from the use of the modified polycarboxylate bonding agent of the Simulate system. The chemical component of bonding of this agent to unconditioned (not acid-etched) enamel is insignificant, since the total bonding strength is only $27.1 \mathrm{kgm} / \mathrm{cm}^{2}$, and is identical to that of the Concise system when tested under similar conditions. The ratio of the bond strength to unetched relative to etched enamel was approximately $19 \%$, similar to that reported in another investigation. ${ }^{13}$
The adhesion of all resin combinations to dentin was slight, as expected. Since acid-etched pretreatment of dentin is not advisable, ${ }^{14}$ clinically significant adhesion of composite restorative resins to dentin awaits further development of chemical bonding systems.

\section{Conclusions}

1. The proposed test method may be used for comparative evaluation of composite resins with good degree of reliability.

2. The four commercial resins that have been studied preformed within clinically acceptable limits.

3. Adhesion to human enamel when a primer is used may be ranked $<$ Adaptic $<$ Concise $<$ Simulate $<$ Nuva-Fil. These differences are not statistically significant, however.

4. Elimination of the intermediary primer does not result in a significant decrease in bond strength for any of the products tested.

5. Bonding to dentin was less than $10 \%$ of that to enamel for all products.

6. No direct evidence was found for chemical bonding to tooth structure by any of the products investigated.

\section{References}

1. Swartz, M.L., and Phillips, R.W.: A Method of Measuring the Adhesive Characteristics of Dental Cement. J Am Dent Ass 50:172-177, 1955.

2. Bowen, R.L.: Adhesive Bonding of Various Materials to Hard Tooth Tissues. I. Method of Determining Bond Strength. J Dent Res 44:690-695, 1965.

3. Mizrahi, E., and Smith, D.C.: Direct Cementation of Orthodontic Brackets of Dental Enamel. Brit Dent J 127:371-375, 1969.

4. Eden, G.T.; Craig, R.G.; and Peyton, F.A.: Evaluation of a Tensile Test for Direct Filling Resins. J Dent Res 49:428-434, 1970. 
5. Laswell, H.R.; Welk, D.A.; and Regenos, J.W.: Attachment of Resin Restoration to Acid Pretreated Enamel. J Am Dent Ass 82:558-563, 1971.

6. Rock, W.P.: The Effect of Etching of Human Enamel Upon Bond Strengths with Fissure Sealant Resins. Arch Oral Biol 19:873-877, 1974.

7. Kemper, R., and Kilian, R.: New Test System for Tensile Bond Testing. Presented at the IADR Mtg., March, 1976. Abstract No. 308, J Dent Res 55:B138, 1976.

8. Bancult, T.A.: Topics in Intermediate Statistical Methods, Vol. I. The Iowa State University Press, Ames, Iowa, P8103, 1968.

9. Siegel, S: Non-Parametric Statistics for the Behavioral Sciences. McGraw Hill, New York, P8202, 1956.

10. Bowen, R.L.: Adhesive Bonding of Various Materials to Hard Tooth Tissues: VI. Forces Developing in Direct Filling Materials During Hardening. J Am Dent Ass 74:439-445, 1967.

11. Mitchem, J.C., and Turner, L.R.: The Retentive Strengths of Acid-Etched Retained Resins. J Am Dent Ass 89:1107-1110, 1974.
12. Jorgensen, K.D., and Shimokobe, H.: Adaptation of Resinous Restorative Materials to Acid-Etched Enamel Surfaces. Scand J Dent Res 83:31-36, 1975.

13. Stanley, H.R.: Going, R.E.; and Chauncey, H.H.: Human Pulp Response to Acid Pretreatment of Dentin and to Composite Restoration. J Am Dent Ass 91:817-825, 1975.

Acknowledgement: The authors wish to recognize the singificant contribution of Dr. Fredrick J. Schoen to this work.

Please direct correspondence to:

Hamdi Mohammed

Professor

Department of Dental Biomaterials

JHMHC Box J-446

University of Florida

Gainesville, Florida 32610 